\title{
Evaluation of next generation emission measurement technologies under repeatable test protocols
}

Clay S. Bell*, Daniel Zimmerle, Timothy Vaughn

Colorado State University, Energy Institute, Fort Collins, CO, 80524

Corresponding author: clay.bell@colostate.edu

\section{Supplementary Material}

\section{Contents}

1. Methane Emission Technology Evaluation Center ....................................................................... 2

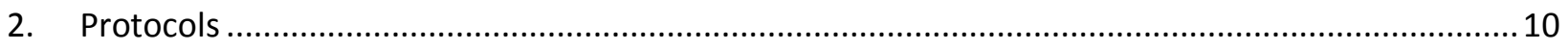

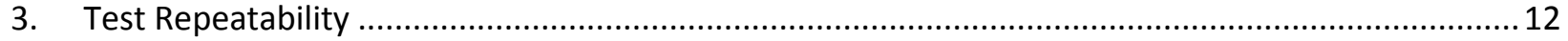

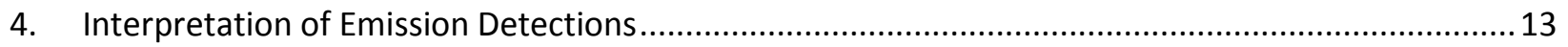

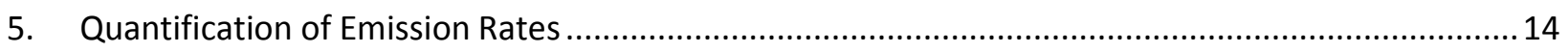




\section{Methane Emission Technology Evaluation Center}

The Methane Emissions Technology Evaluation Center (METEC) was developed at Colorado State University's (CSU) Foothills Campus as the ARPA-E MONITOR field test site. METEC is located west of Fort Collins, Colorado and is isolated from other potentially interfering sources of methane (livestock, oil and gas operations, wetlands, etc.). The facility design was developed to provide testing conforming to the ARPA-E MONITOR program metrics:

- Detect emission sources of $6 \mathrm{SCFH}$ and greater

- Locate emission sources within $1 \mathrm{~m}$ on a $10 \mathrm{~m}$ x $10 \mathrm{~m}$ production well pad

- Quantify emission sources within $+/-20 \%$ of the actual emission rate

Additional features were included in the design to provide testing on larger footprint pads and to extend the use of the facility to other natural gas sectors. Figure 1 provides a snapshot view of the entire facility, with major systems marked. METEC includes two $10 \mathrm{~m} \times 10 \mathrm{~m}$ well pads (consistent with the ARPA-E MONITOR metric), three larger well pads representing a variety of equipment configurations and layouts, a small mock compressor station, and a pipeline test bed. In total the facility has 198 aboveground controlled emission release points and 52 underground release points. Up to 60 emission points can be controlled simultaneously, with time-varying release rates throughout the site. The facility is operated from a control room situated on the east side of the site which also provides office and meeting space for staff and visitors. A roadway around the site provides access for measurements by mobile laboratories and easy movement throughout the facility for installation of stationary measurement equipment. Metrological data including wind speed and direction, ambient temperature, barometric pressure, and relative humidity are collected on a $6 \mathrm{~m}$ tower at the west end of the facility.

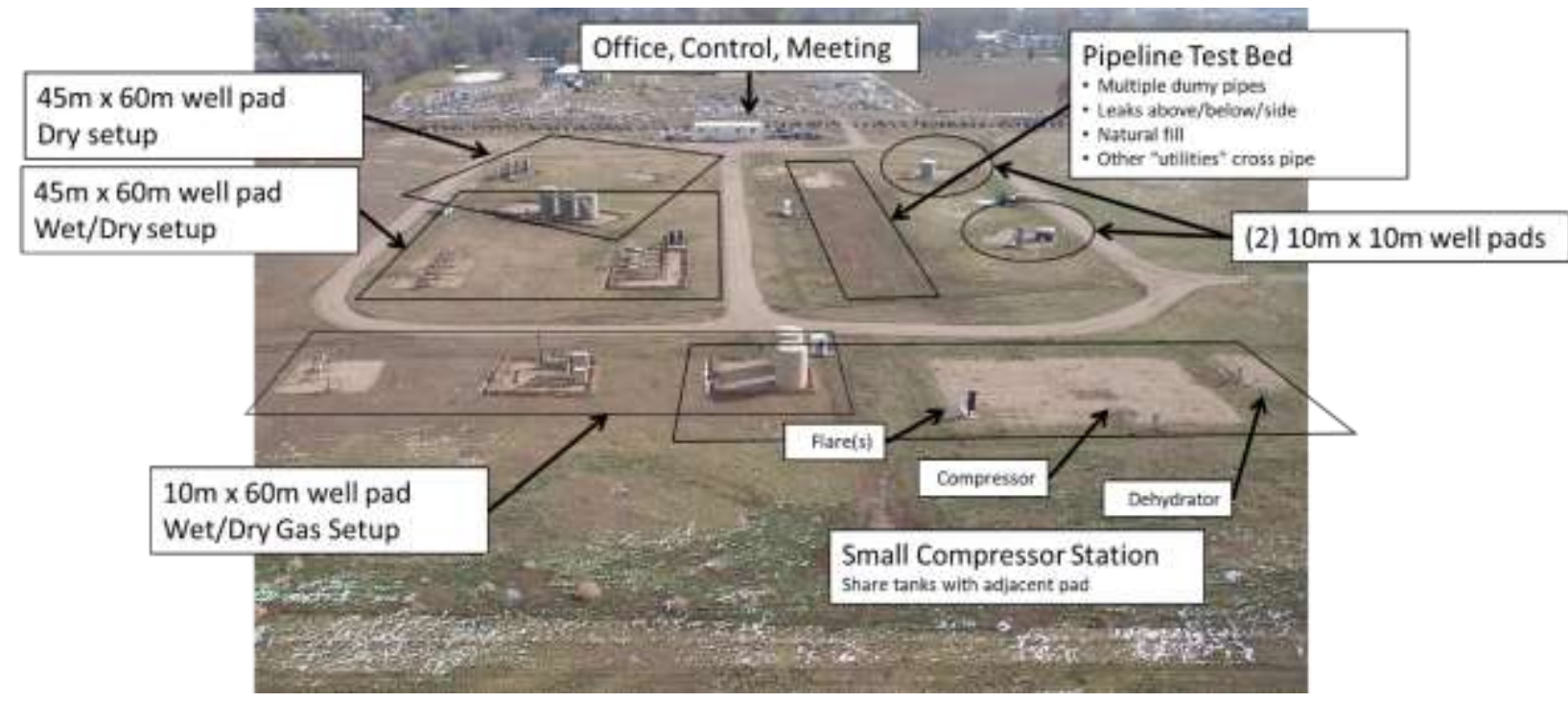

Figure 1. METEC layout from an aerial perspective.

METEC includes 12 acres within its fence line and emulates well pads, a small compressor station, and underground pipelines laid out in 7 distinct "Pads" covering approximately 7 acres (Figure 2). Since most next-generation leak detection solutions detect leaks using downwind transport of emissions, the layout of the facility was designed to recreates the wind fields by using real oil and gas equipment situated in 
Evaluation of next generation emission measurement technologies under repeatable test protocols - Supplementary Material -

realistic upstream site configurations. To eliminate the potential for bias in testing due to a single pad orientation relative to predominate wind directions, the facility design rotated Pad 2 and Pad 5 by $45^{\circ}$ and $30^{\circ}$ respectively. Pads $1-5$ were used in the experiments discussed in this paper.

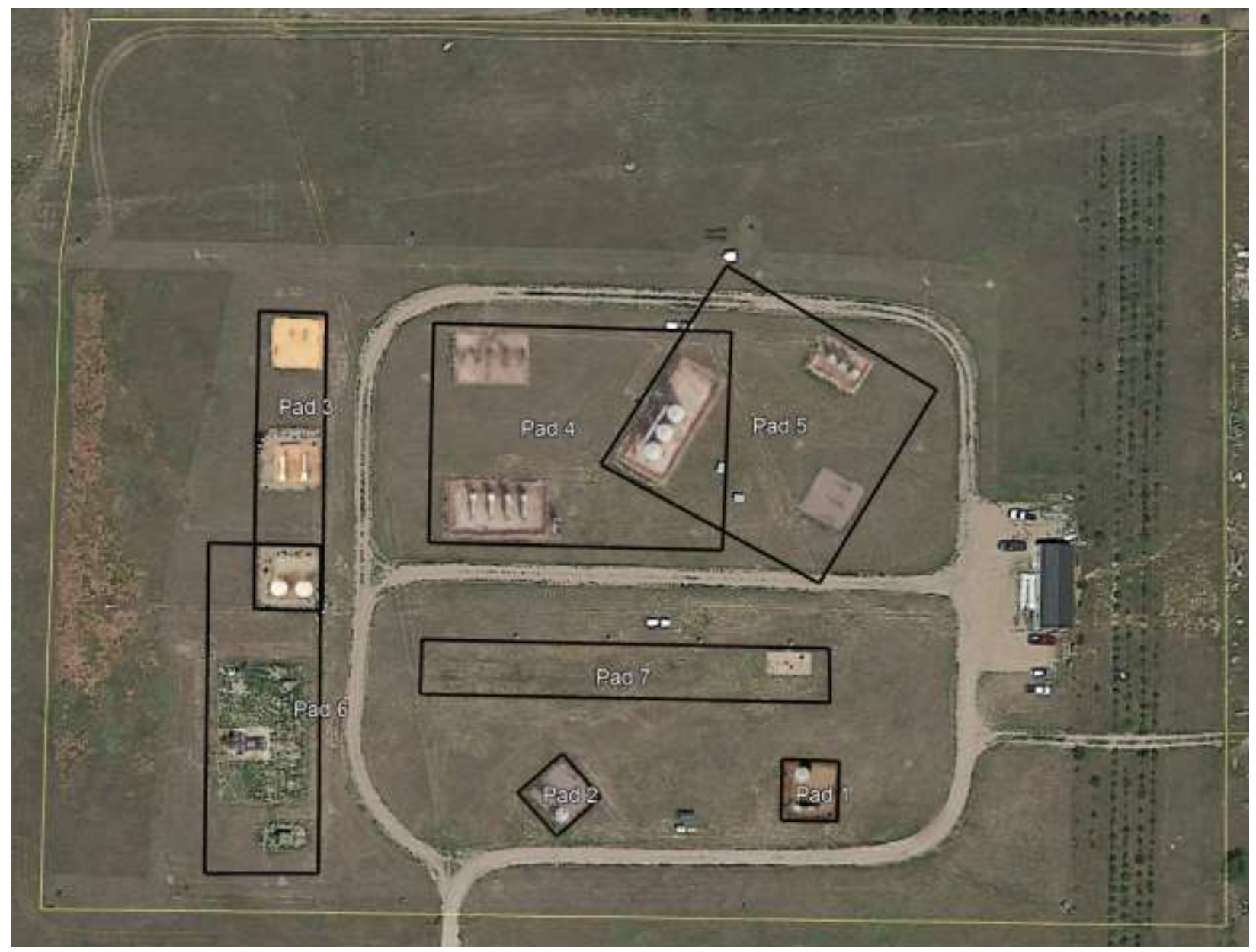

Figure 2: METEC fenceline (yellow) and pad layout (black) illustrated on Google Earth.

\section{Pad 1}

Pad 1 is a $10 \mathrm{~m} \times 10 \mathrm{~m}$ mock production well pad. The pad is outfitted with one wellhead, one horizontal separator, and one 150 bbl liquid storage tank. 


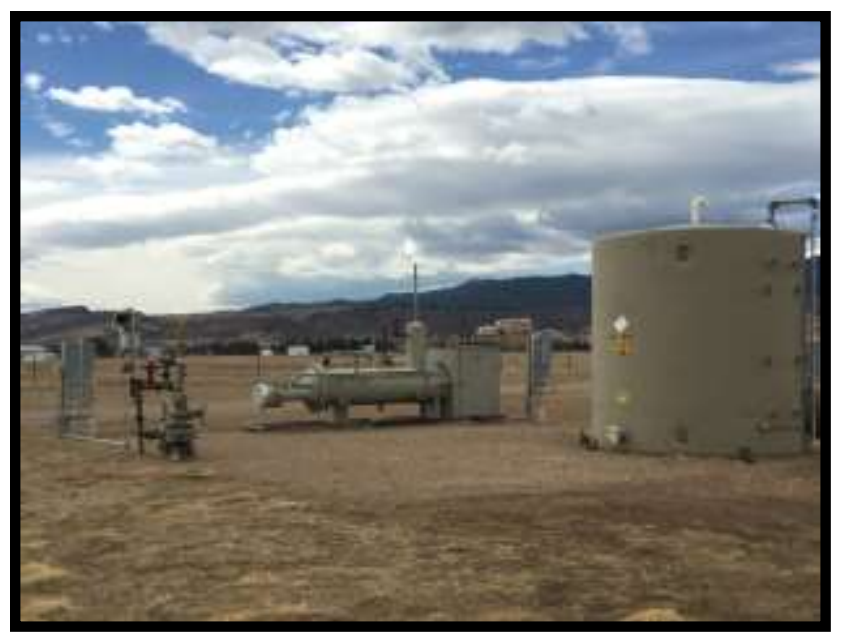

Figure 3: METEC Pad $1-10 \mathrm{~m} \times 10 \mathrm{~m}$ production well pad with 1 wellhead, 1 horizontal separator, and 1 liquid storage tank

\section{Pad 2}

Pad 2 is a $10 \mathrm{~m} \times 10 \mathrm{~m}$ mock production well pad. The pad is outfitted with one wellhead, one vertical separator, and one $70 \mathrm{bbl}$ produced water storage tank.

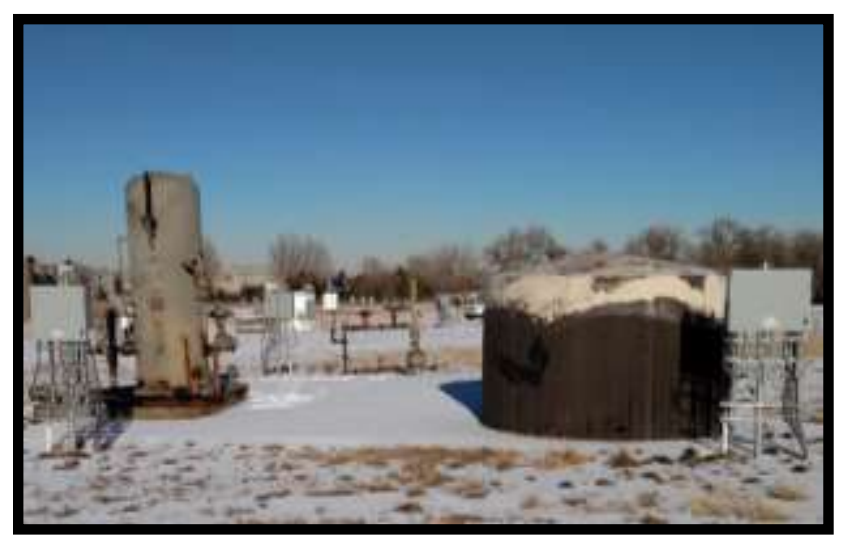

Figure 4: METEC Pad 2-10m x 10m production well pad with 1 wellhead, 1 vertical separator, and 1 liquid storage tank

\section{Pad 3}

Pad 3 is a $60 \mathrm{~m} \times 10 \mathrm{~m}$ mock production well pad. Instead of grading and graveling the whole area, the pad is composed of three $10 \mathrm{~m} \times 10 \mathrm{~m}$ subpads, each separated by $15 \mathrm{~m}$ and arranged North to South. This layout is intended to provide small $10 \mathrm{~m} \times 10 \mathrm{~m}$ testing facilities conforming to the ARPA-E MONITOR metric, however when viewed together represent a more realistic size production well pad. The overall pad is outfitted with three wellheads, two horizontal separators, and two 300 bbl liquid storage tanks. Earthen berms are included around the separation equipment and liquid storage tanks to provide the effect on dispersion from secondary containment systems found at real oil and gas facilities. 


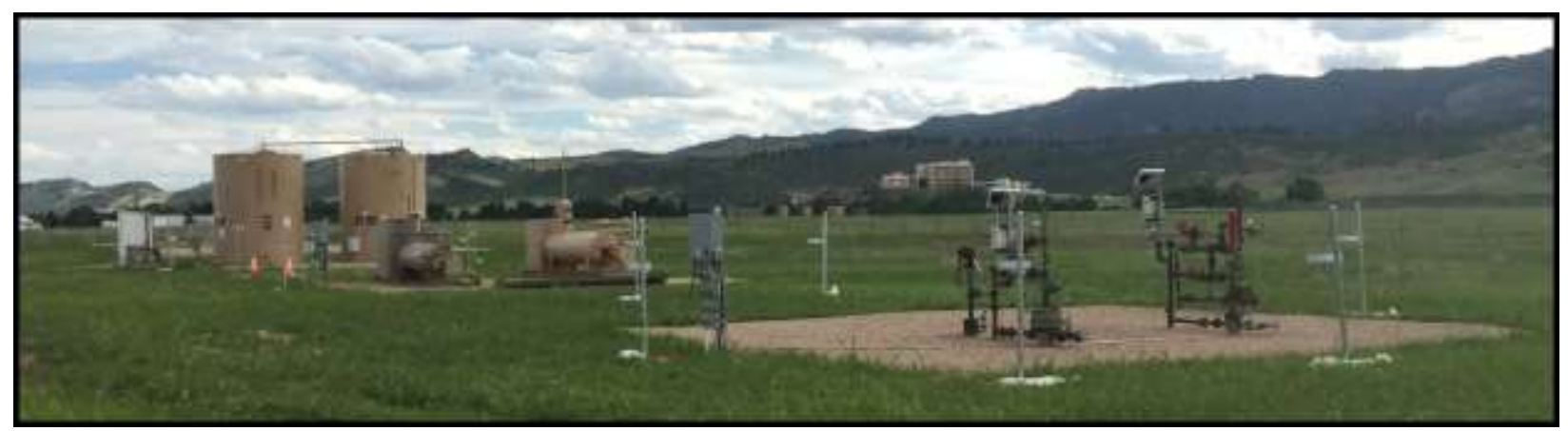

Figure 5: METEC Pad $3-60 m \times 10 m$ production well pad with 3 wellheads, 2 horizontal separators, and 2 liquid storage tanks

\section{Pad 4}

Pad 4 is a $60 \mathrm{~m} \times 45 \mathrm{~m}$ mock production well pad. Instead of grading and graveling the whole area, Pad 4 is composed of three subpads of different sizes. The pad is outfitted with five wellheads on a $10 \mathrm{~m} \times 15 \mathrm{~m}$ subpad, four horizontal separators on a $10 \mathrm{~m} \times 20 \mathrm{~m}$ subpad, and three $300 \mathrm{bbl}$ liquid storage tanks with a catwalk on a $10 \mathrm{~m} \times 25 \mathrm{~m}$ subpad. This layout is intended to provide testing facilities which are more representative of upstream production sites in both size, equipment spacing and complexity. Earthen berms are included around the separation equipment and liquid storage tanks to provide the effect on dispersion from secondary containment systems found at real oil and gas facilities.

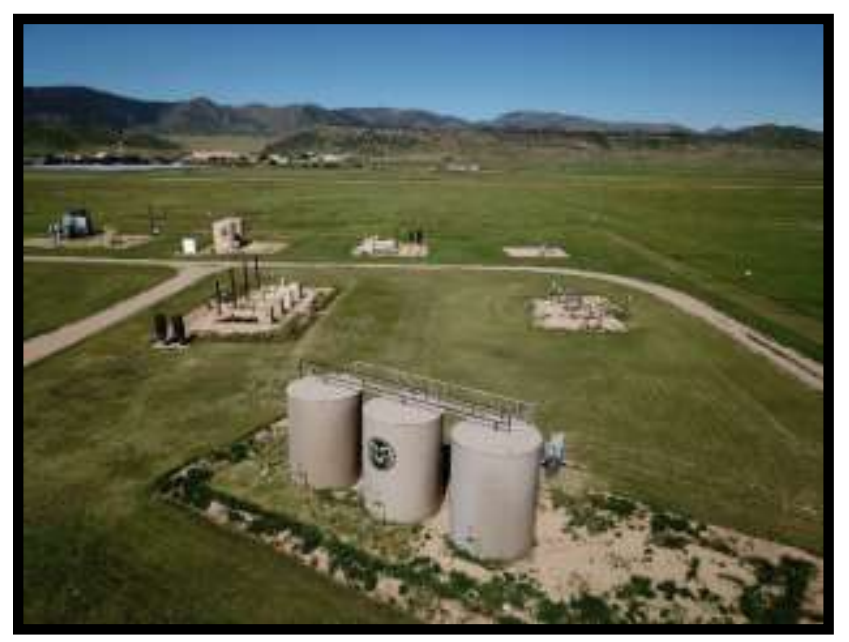

Figure 6: METEC Pad 4 - Aerial view of Pad 4 in foreground with 5 wellheads, 4 horizontal separators, and 3 liquid storage tanks. Pad 3 in background

\section{Pad 5}

Pad 5 is a $60 \mathrm{~m} \times 45 \mathrm{~m}$ mock production well pad. Instead of grading and graveling the whole area, Pad 5 is composed of three subpads of different sizes. The pad is outfitted with three wellheads on a $10 \mathrm{~m} x$ $10 \mathrm{~m}$ subpad, three vertical separators on a $10 \mathrm{~m} \times 15 \mathrm{~m}$ subpad, and shares the three $300 \mathrm{bbl}$ liquid storage tanks used in Pad 4. This layout is intended to be similar to Pad 4, with a different variety of equipment found in other production regions. Earthen berms are included around the separation equipment and liquid storage tanks to provide the effect on dispersion from secondary containment systems found at real oil and gas facilities. 


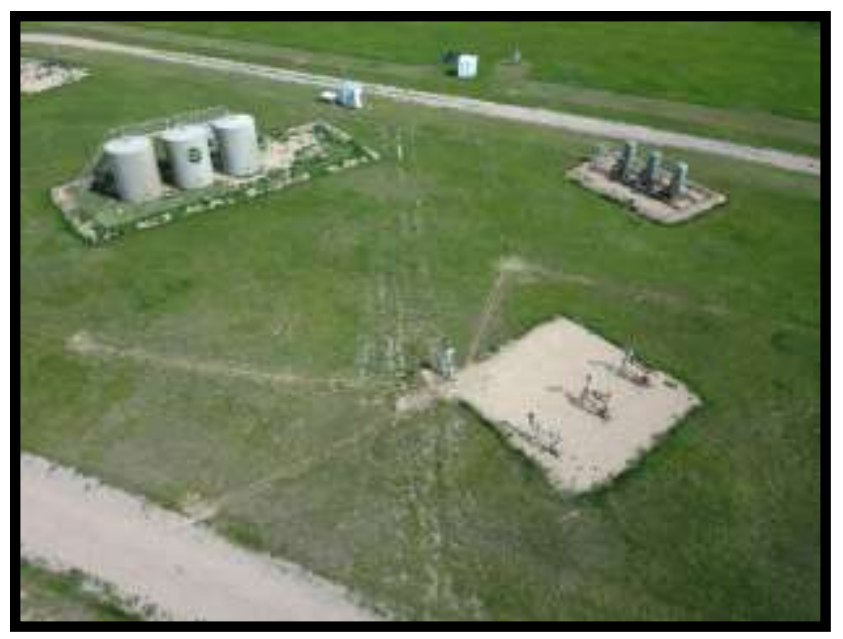

Figure 7: METEC Pad 5-Aerial view of Pad 4 with 3 wellheads, 3 vertical separators, and 3 liquid storage tanks. Pad 3 in background

\section{Gas Control and Metering System}

The gas control and metering system is the backbone of the METEC facility. A simplified P\&ID for a gas control and metering system is shown in Figure 8. A set of Compressed Natural Gas (CNG) cylinders which can be refilled using standard components from CNG vehicle fuel systems are installed at each of four Gas Supply Houses (GSH) throughout the facility (Figure 8 section (1)). A CNG vehicle regulator supplies gas from the cylinder outlets to the gas metering system at a fixed pressure of $150 \mathrm{psig}$. The CNG regulator is heated using a pumped glycol loop to prevent the component from becoming superchilled due to the Joule Thompson cooling from the expanding gas. A separate inlet exists to allow the system to be supplied from a set of gas cylinders with a specified composition if required for a given series of tests. An adjustable regulator further regulates the gas pressure between 0-120 psig, reducing drift and improving flow stability. The regulated gas flow is then divided to multiple branches, each of which is metered (Figure 8 section (2)). Several meters of different spans are selectable through manual valves and allow the operator to measure the gas flowrate using a meter most appropriate for the emission rates in a given test. The metered branches are then divided and deliver gas from the gas supply houses to a set of emission controllers at the pads (Figure 8 section (3)). A set of pressure regulators allows the pressure of each controller to be adjusted independently of the others. The controllers are located near the equipment on each pad and use a series of solenoid operated valves and precision orifices to provide independent control of the emission rate of each emission point (Figure 8 section (4)). Emission points on the pad can be connected to the controller outlets, allowing many configurations to be implemented throughout the site (Figure 8 section (5)). Pressures and temperatures are measured throughout the system. 
Evaluation of next generation emission measurement technologies under repeatable test protocols - Supplementary Material -

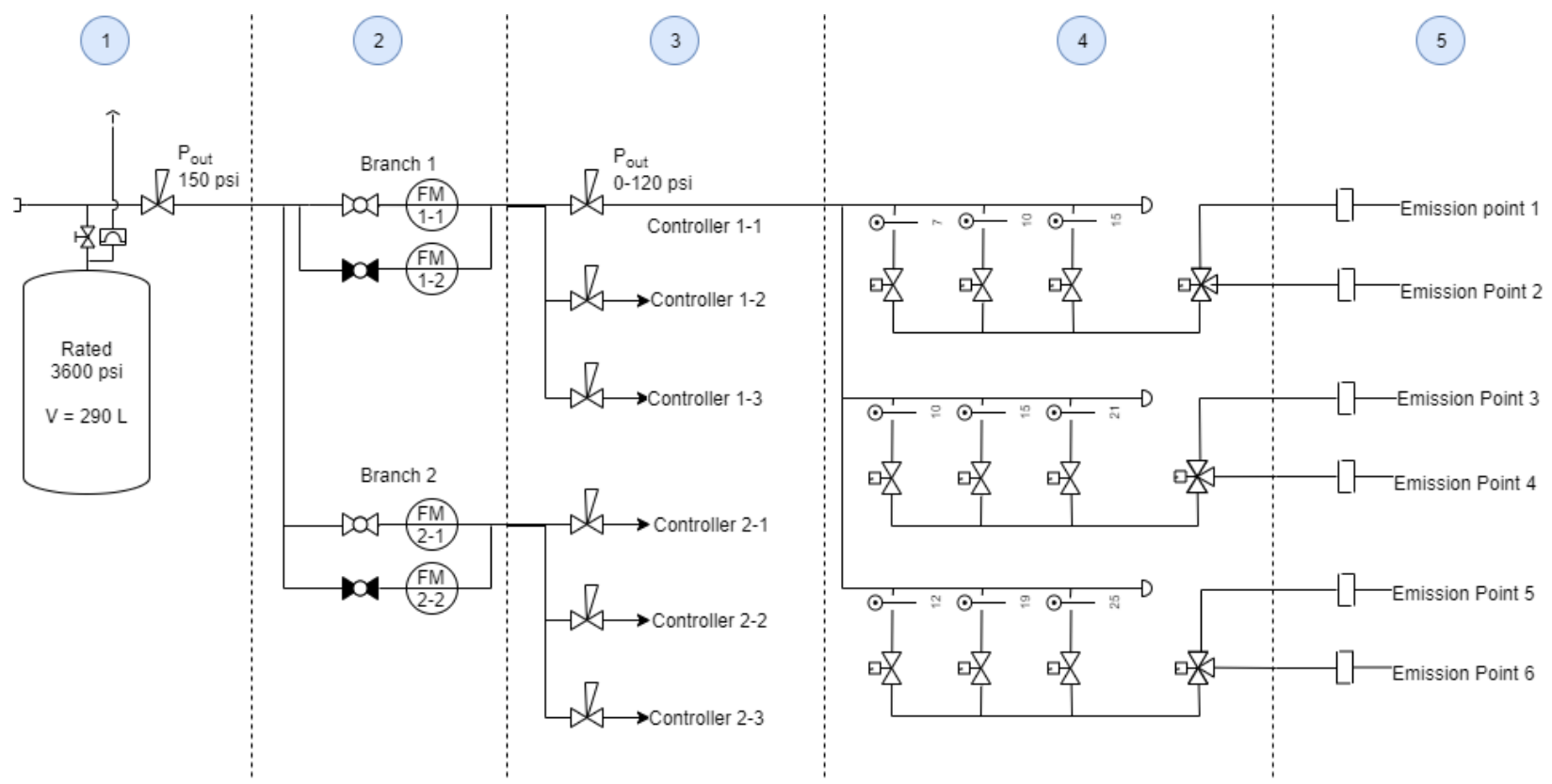

Figure 8: Simplified P\&ID of gas control and metering system

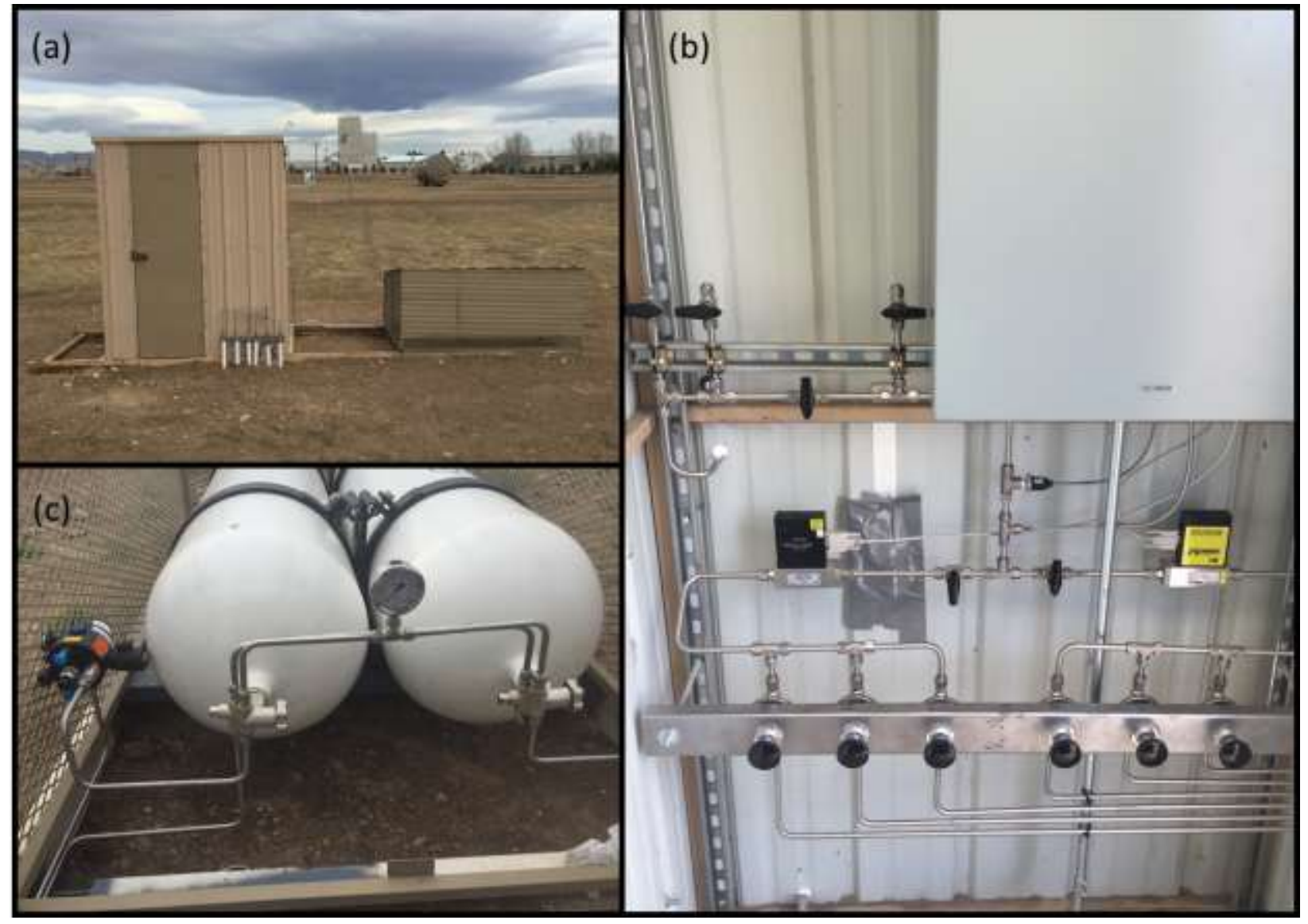

Figure 9: Gas control and metering. (a) Exterior of Gas Supply House at METEC, (b) Interior with flow metering and controller branch regulation, (c) Refillable CNG cylinders and regulator are primary supply for each gas supply house. 
The span of all flowmeters initially installed throughout the site were 0-75 Ipm with an accuracy of +/$1 \%$ span (+/- $0.75 \mathrm{lpm})$. Additional flowmeters with span of $0-7.5 \mathrm{lpm}$ and accuracy of $+/-1 \%$ span (+/$0.075 \mathrm{lpm}$ ) were installed to improve the accuracy of measurements during tests at low emissions rates. Finally, additional flow meters with span of 0-150 lpm and 0-375 lpm with accuracies of $+/-1.5 \%$ span (+/-2.25 lpm and +/-5.63 lpm respectively) were installed to extend the capabilities of the facility by providing the ability to meter larger emission rates.

Each flow controller, Figure 10, consists of an inlet manifold, and either two or three rows of solenoid actuated gas shutoff valves, as seen in Figure 8 section (4). The valves in a single row flow into an outlet manifold which is directed by a final solenoid actuated directional valve to one of two emission locations. Each gas shutoff valve is fed from the inlet manifold by a different size precision orifice. The precision orifices were selected to produce a nearly linear set of emission rates at a fixed pressure. By adjusting the pressure setpoint at the inlet manifold, the span of each row of the controller is adjusted. This allows the controller to achieve finer steps across a smaller span when operating at a low pressure, and coarser steps across a larger span when operating at a high pressure using the same series of orifices. By including multiple rows of valves, each controller may control multiple emission points simultaneously. A total of 21 controllers are installed throughout the facility. The orifices in most controllers were selected to allow emissions between 0 and 150 SCFH to be simulated. This allows lower emission rates around the ARPA-E MONITOR metric (6 SCFH) to be controlled to simulate fugitive emission sources, while simultaneously controlling other emission sources at higher emission rates to simulate normal venting from pneumatic devices.

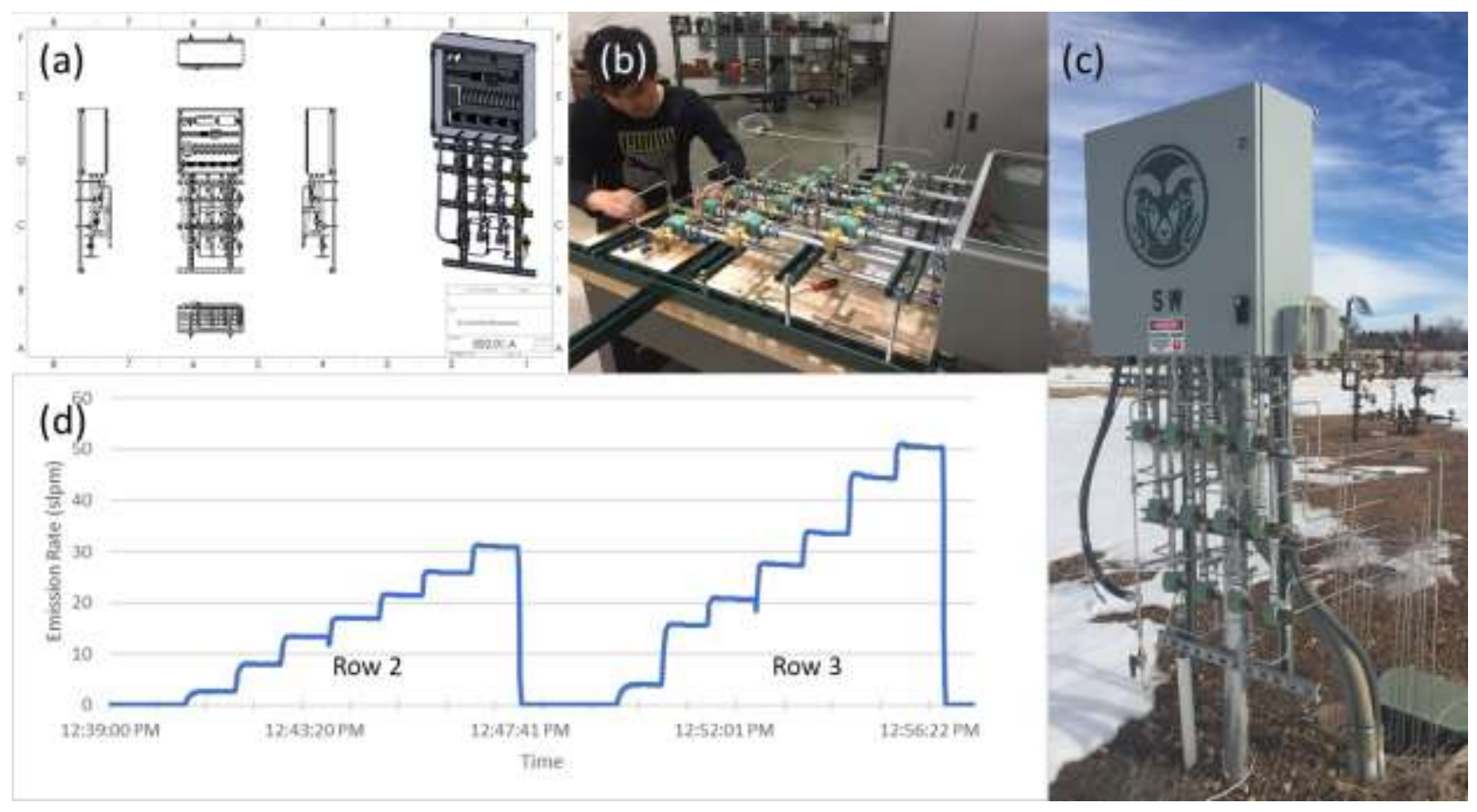

Figure 10: METEC flow controller. (a) CAD drawing for prototype controller, (b) assembling prototype controller, (c) Finished controller installed for Pad 5 well heads, (d) Demonstration data of emission rate control using orifices installed in rows 2 (0-30 slpm) and 3 (0-50 slpm) on Pad 5 well heads controller operating at 100 psia setpoint. 


\section{- Supplementary Material -}

Emissions throughout the entire facility are controlled from a central computer. The METEC control software allows the operator to actuate any given valve throughout the facility, and monitor readings from flowmeters, pressure transducers, and thermocouples throughout the system. Data including metrological data, system temperatures, pressures, flowrates, and valve positions are collected at $1 \mathrm{~Hz}$.

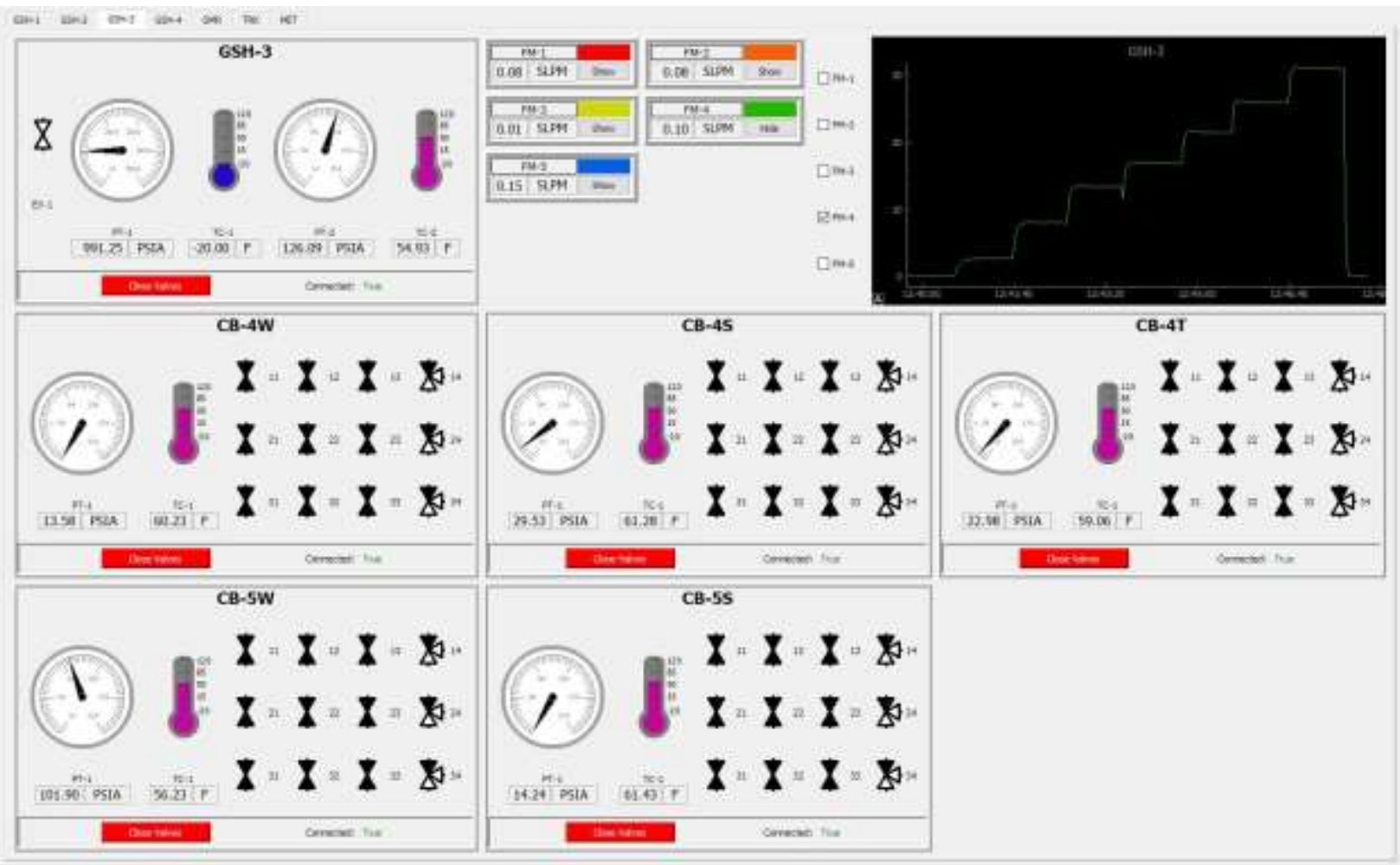

Figure 11: Data acquisition and control system implemented in python.

\section{Emission Points}

A total of 198 above-ground controlled emission release points and 52 underground release points exist throughout the facility. Above ground emission release points are designed to coincide in location with emission sources observed in the field, including both intentional emission sources such as pneumatic venting, and fugitive sources or "leaks". Where possible gas is routed from the controller outlet to the emission location in a concealed fashion using existing small diameter piping on the exterior of equipment, or inside the equipment itself as shown in Figure 12. By concealing the gas lines and engineering the leak locations "blind" testing can be performed at METEC for not only remote monitoring systems, but also traditional LDAR methods where a human operator performs a survey of the facility. 


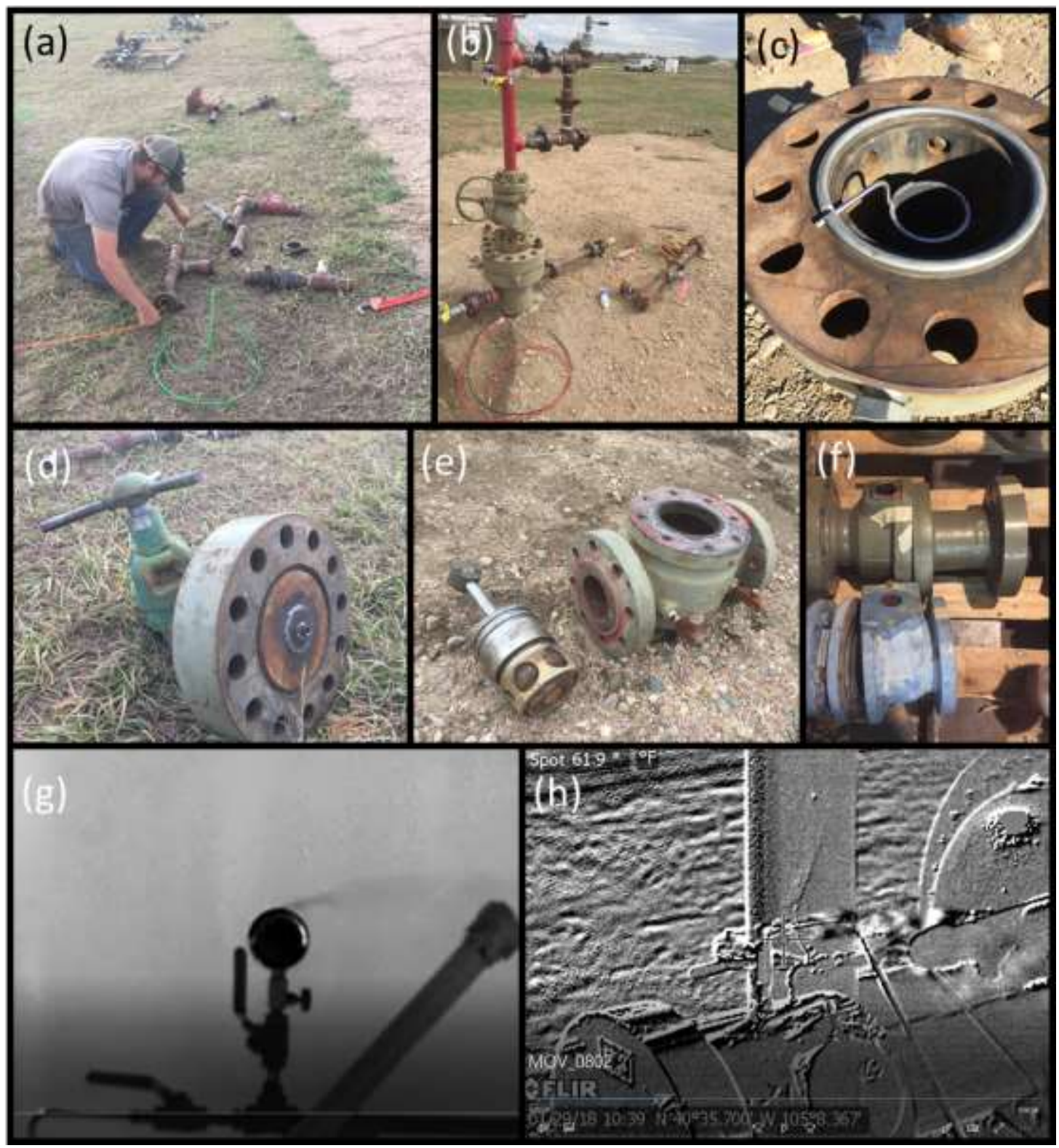

Figure 12:METEC Emission Points. (a)-(d) Routing lines to emission points inside wellheads during assembly, (e)-(f) Valve bodies disassembled to make controlled leaks from valve stems, $(g)$-( $h$ ) controlled emissions from a pressure gauge and a fitting at METEC viewed through an OGI camera.

\section{Protocols}

Two different protocol were developed to better support testing of two solution archetypes: survey solutions and continuous monitoring solutions. The survey protocol required solutions to move from pad to pad throughout each test day. The continuous monitoring protocol required systems to set up to monitor only METEC Pad 4 throughout the entire series of tests. Under each protocol 15 experiments were developed at three levels of complexity designed to extend testing from simple single source characterization to more realistic scenarios where multiple sources may be present and intermittent emissions from sources such as pneumatic actuators are included. 
Evaluation of next generation emission measurement technologies under repeatable test protocols - Supplementary Material -

\section{Survey Protocol}

To evaluate survey methods the following procedure was followed:

1. The METEC operator selected and assigned a pad to the performer.

2. The METEC operator ensured operating pressure was at the setpoint and started a predefined emissions scenario on the assigned pad.

3. The performer was informed they may begin their measurement. Performers were not informed of the number of emission sources, the rates of emission sources, the location of emission sources, or the complexity level of the test. No time-limit was allotted for an individual test, though teams were required to complete the full round of 15 tests in the five-day period.

4. The performer completed their method to "survey" the assigned the pad.

5. The performer informed the METEC operator when they had completed their assessment of emissions from the pad.

6. The METEC operator stopped the emissions scenario on the assigned pad.

7. The METEC operator assigned the next pad and steps 2-7 were repeated until all 15 tests were completed.

\section{Continuous Monitoring Protocol}

To evaluate continuous monitoring solutions the following procedure was followed:

1. The continuous monitoring system was installed at METEC to assess emissions on pad 4.

2. The METEC operator started a predefined emissions scenario on the pad.

3. The measurement team was informed they may begin their measurement. Measurement teams were not informed of the number of emission sources, the rates of emission sources, the location of emission sources, or the complexity level of the test. The measurement team was allowed to collect data for an allotted amount of time. The time allotted was determined by the total testing period the measurement team had reserved $(2 \mathrm{~h} /$ test for a five-day period, $4 \mathrm{~h} /$ test for a ten-day period).

4. After the allotted time had elapsed, the METEC operator stopped the emissions scenario on the assigned pad.

5. The METEC operator selected the next emission scenario and steps 2-5 were repeated until all 15 predefined scenarios were completed.

\section{Data Reporting}

A standardized format for reporting emission assessments was provided by METEC to each measurement team. The same format was used for the basin survey protocol and the continuous monitoring protocol. The spreadsheet allowed the measurement team to report the location (pad, equipment type, equipment ID, and gps coordinates) and flowrate estimate for each emission source observed during each test. The spreadsheet was provided with an accompanying. $\mathrm{kml}$ file which included a standardized $0.25 \mathrm{~m}$ grid overlaid on aerial imagery of METEC and referenced to a survey point on the facility. This grid overlay, Figure 13, enabled systems without GPS to report latitude and 
longitude of emission locations at a $0.25 \mathrm{~m}$ resolution for use in the $2 \mathrm{D}$ and $3 \mathrm{D}$ distance calculations. Distance calculations were based on the difference between reported coordinates and actual emission locations at METEC measured using a GPS antenna with RTK correction.

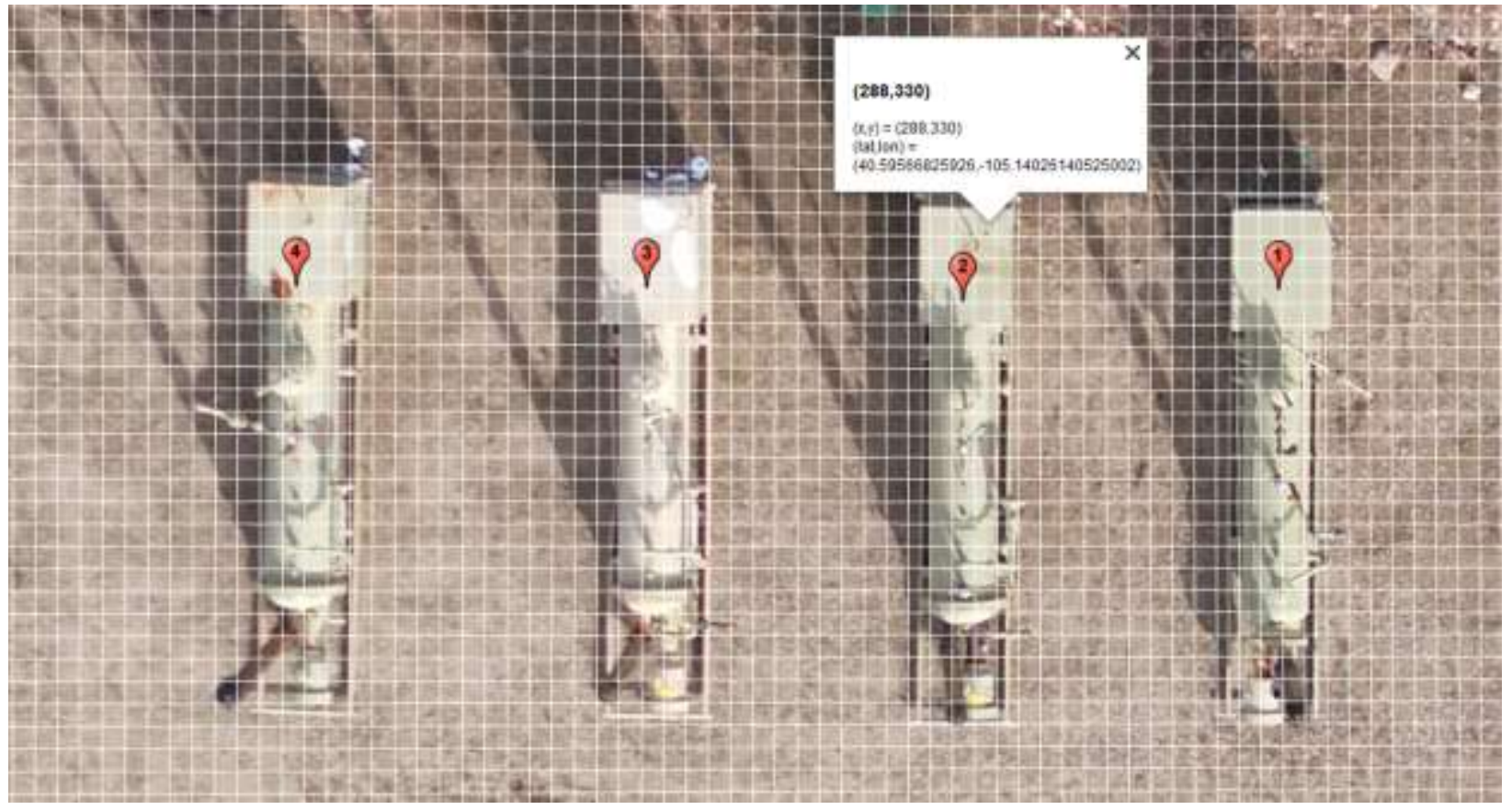

Figure 13: Round 2 grid overlay for pad 4 separators allowing equipment IDs (red ballons) and GPS coordinates of detected emission sources to be reported.

Traditional approaches to leak detection using a handheld instrument often physically tag detected emission sources using caution tape or ribbon to mark them for repair. In this series of tests teams did not tag emission sources, and were instead required to report detections using the standard format described above. This may be a disadvantage in the testing protocol compared to mobile and continuous monitoring methods which perform localization of emission sources relative to an on-board gps unit.

\section{Test Repeatability}

Tests scenarios including the emission source locations and emission rates were reproduced for each tester. Figure 14 shows test to test repeatability for the survey solutions and Figure 15 for the continuous monitoring solutions. Variation in emission rate for an individual emission source results from variation in the manually adjusted pressure setpoint for the experiment and from variation in the gas composition over the course of testing. 


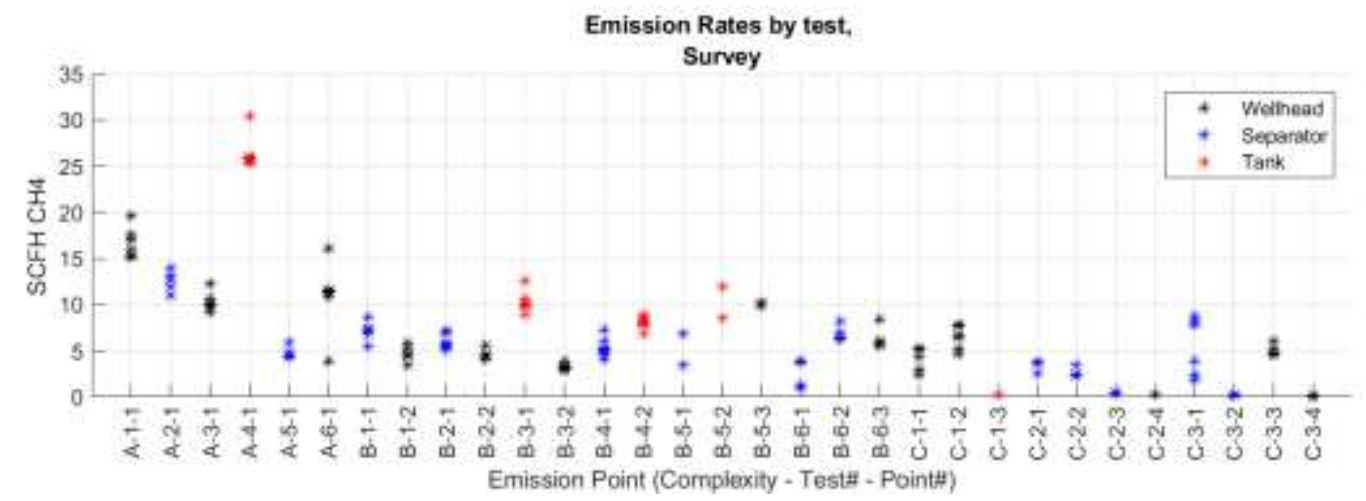

Figure 14: Emission rates for each emission source present in survey testing. Variation in methane emission rate for a given source is attributed to manually adjusted pressure setpoints and gas compositions.

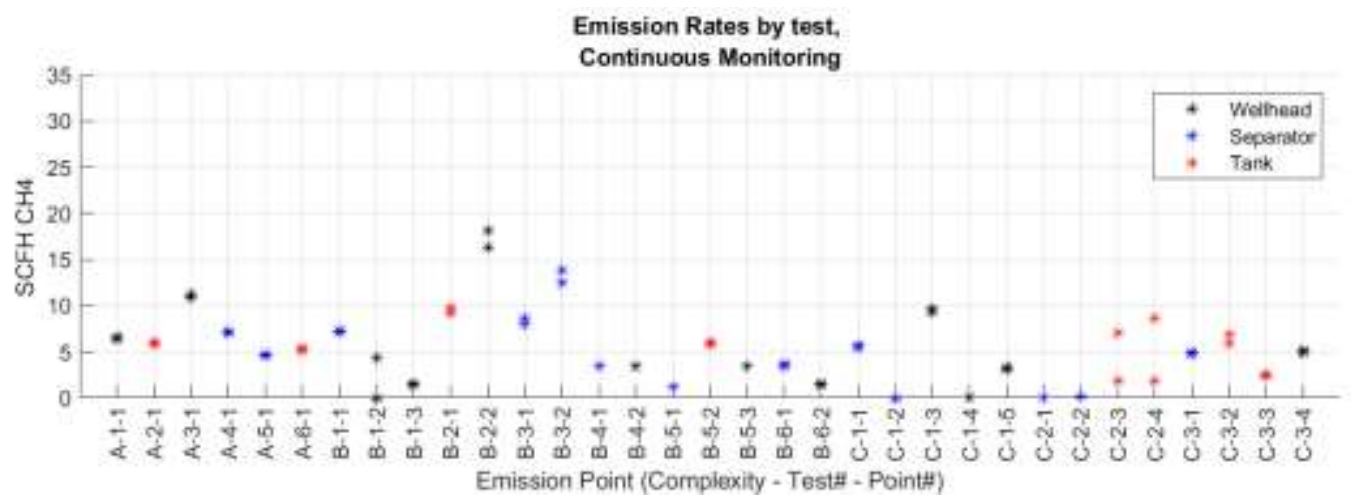

Figure 15: Emission rates for each emission source present in continuous monitoring testing. Variation in methane emission rate for a given source is attributed to manually adjusted pressure setpoints and gas compositions.

\section{Interpretation of Emission Detections}

Equipment IDs reported by performers were used to determine whether emission sources were detected. Each reported emission source was interpreted as an equipment detection, a group detection, or a false positive as illustrated in Figure 16. Controlled emission sources which were not attributed to a reported emission source were identified as a non-detection. Some emission detections in the SI Data Tables are labeled as "Multi Detect". This represents a special case used when two emission sources were within $1 \mathrm{~m}$ of one another and it was impossible to discern which was detected. In this case both sources were marked as a detection using the "Multi Detect" code. In the analysis, we consider MultiDetect equivalent to Equipment Detect. 
Evaluation of next generation emission measurement technologies under repeatable test protocols - Supplementary Material -

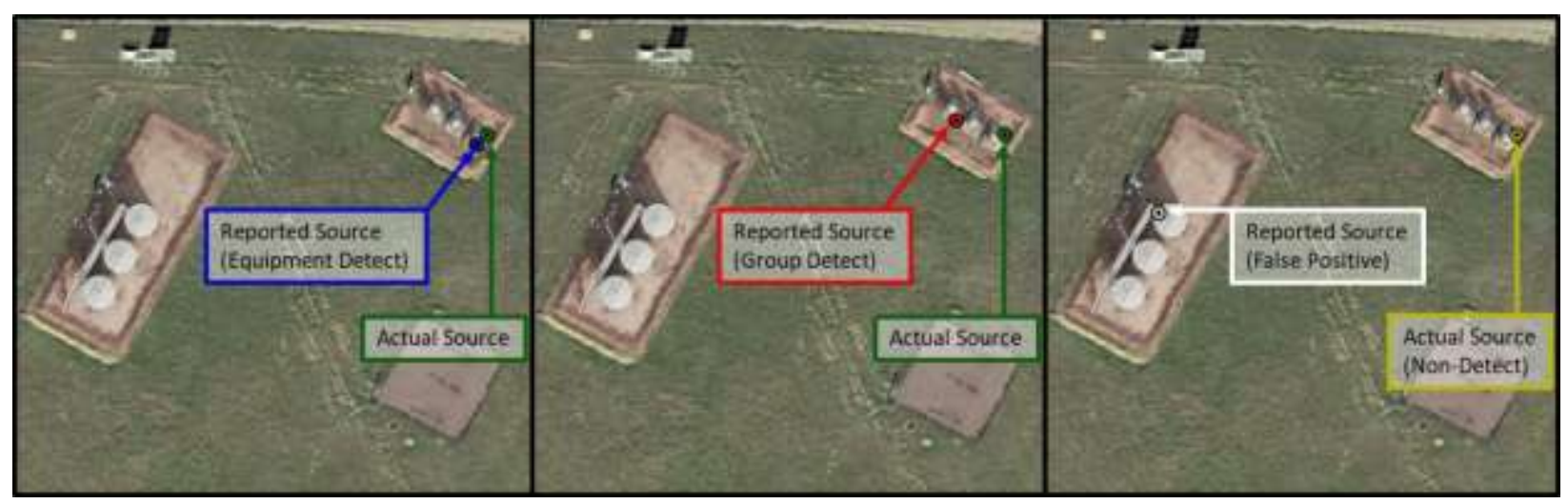

Figure 16: Interpretation of emission detections on Pad 5. A reported source and actual source on the same unit of equipment interpreted as an equipment detect (left), a reported source and actual source on the same group of equipment but different equipment units interpreted as a group detect (center), and a reported source and actual source on different equipment groups interpreted as a false positive and a non-detect (right).

\section{Quantification of Emission Rates}

Reported emission rates with uncertainty estimates (if reported by the performer) are plotted versus metered emission rates with uncertainty estimates in Figure 17. Overall uncertainty in the metered emission rate is shown at $95 \%$ confidence level and includes uncertainties due to meter accuracy, flow stability, and gas composition. Mobile quantification estimates averaged $17 \%$ higher than metered emission rates, while estimates from continuous monitoring systems averaged $167 \%$ higher than metered emission rates. 


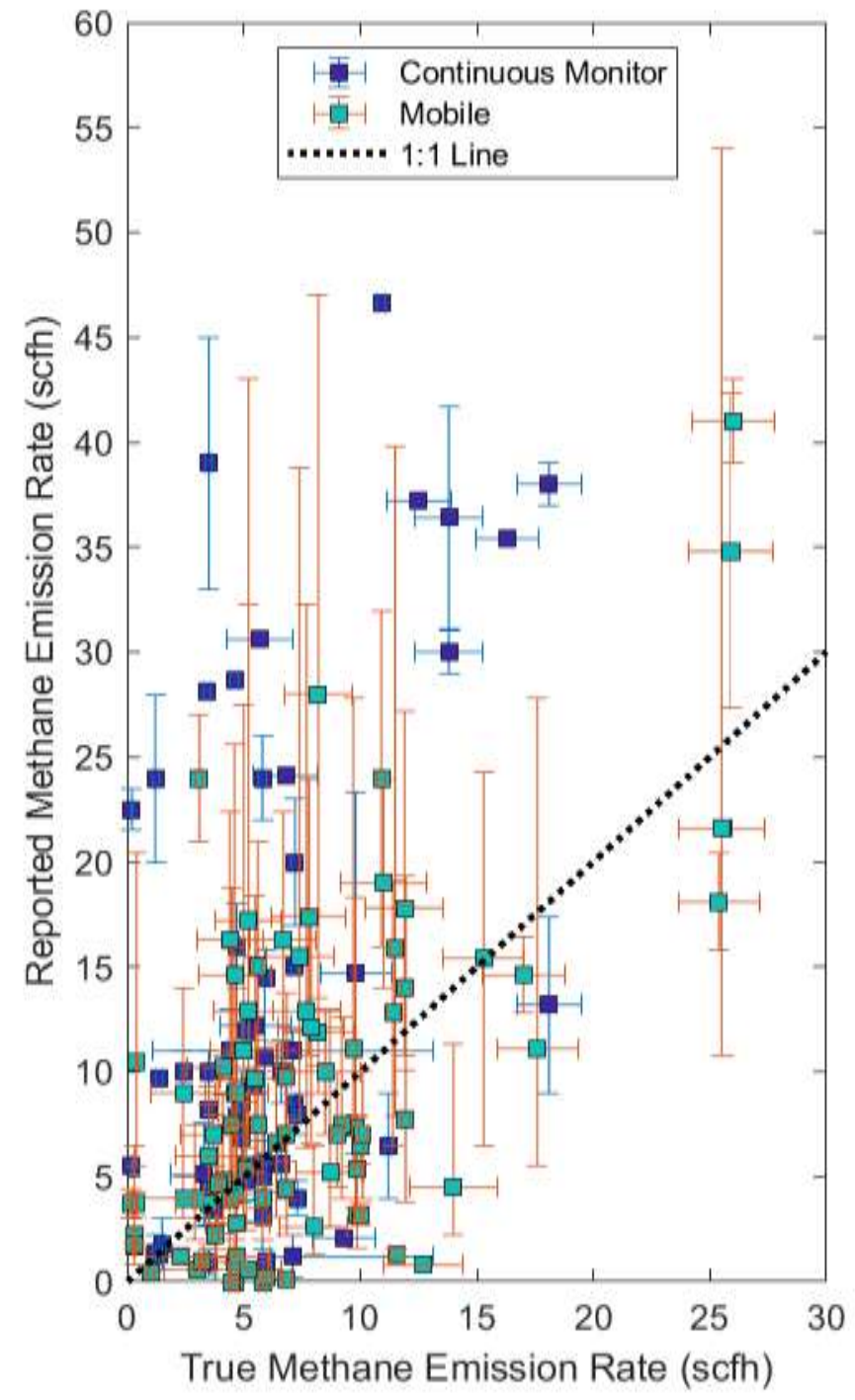

Figure 17: Reported emission rate versus metered emission rate. 